\title{
Coupled Coincidence Point Results for Mixed $(G, S)$-Monotone Mapping and Applications
}

\author{
Habib Yazidi* \\ Department of Mathematics, Tunis College of Sciences and Techniques, University of Tunis, 5 Avenue Taha Hussein, BP 59, Bab \\ Mnara, Tunis, Tunisia
}

Received: 17 Aug. 2013, Revised: 19 Nov. 2013, Accepted: 20 Nov. 2013

Published online: 1 Jul. 2014

\begin{abstract}
We introduce the concept of mixed $(G, S)$-monotone mappings and prove coupled coincidence point theorems for such mappings satisfying a nonlinear contraction involving altering distance functions. Presented theorems extend, improve and generalize the recent results of Harjani, López and Sadarangani [J. Harjani, B. López and K. Sadarangani, Fixed point theorems for mixed monotone operators and applications to integral equations, Nonlinear Anal. 74 (2011) 1749-1760] and other existing results in the literature. As application, we present an existence theorem for solutions to a system of nonlinear integral equations.
\end{abstract}

Keywords: Coincidence point, $(G, S)$-monotone mapping, ordered set, altering distance, integral equations.

\section{Introduction and preliminaries}

Fixed point problems of contractive mappings in metric spaces endowed with a partially order have been studied by many authors (see [1]-[17]). Bhaskar and Lakshmikantham [3] introduced the concept of a coupled fixed point and studied the problems of a uniqueness of a coupled fixed point in partially ordered metric spaces and applied their theorems to problems of the existence of solution for a periodic boundary value problem. In [8], Lakshmikantham and Ćirić established some coincidence and common coupled fixed point theorems under nonlinear contractions in partially ordered metric spaces. Very recently, Harjani, López and Sadarangani [7] obtained some coupled fixed point theorems for a mixed monotone operator in a complete metric space endowed with a partial order by using altering distance functions. They applied their results to the study of the existence and uniqueness of a nonlinear integral equation. Now, we briefly recall various basic definitions and facts.

Definition 11(see Bhaskar and Lakshmikantham [3]). Let $(X, \preceq)$ be a partially ordered set and $F: X \times X \rightarrow X$. Then the map $F$ is said to have mixed monotone property if $F(x, y)$ is monotone non-decreasing in $x$ and is monotone non-increasing in $y$, that is,

$$
x_{1} \preceq x_{2} \text { implies } F\left(x_{1}, y\right) \preceq F\left(x_{2}, y\right) \text { for all } y \in X
$$

and

$y_{1} \preceq y_{2}$ implies $F\left(x, y_{2}\right) \preceq F\left(x, y_{1}\right)$ for all $x \in X$.

The main result obtained by Bhaskar and Lakshmikantham [3] is the following.

Theorem 11(see Bhaskar and Lakshmikantham [3]). Let $(X, \preceq)$ be a partially ordered set and suppose there is a metric $d$ on $X$ such that $(X, d)$ is a complete metric space. Let $F: X \times X \rightarrow X$ be a mapping having the mixed monotone property on $X$. Assume that there exists $k \in[0,1)$ such that

$$
\begin{aligned}
& d(F(x, y), F(u, v)) \leq \frac{k}{2}[d(x, u)+d(y, v)] \\
& \text { for each } u \preceq x \text { and } y \preceq v .
\end{aligned}
$$

Suppose either $F$ is continuous or $X$ has the following properties:

(i)if a non-decreasing sequence $x_{n} \rightarrow x$, then $x_{n} \preceq x$ for all $n$,

(ii)if a non-increasing sequence $x_{n} \rightarrow x$, then $x \preceq x_{n}$ for all $n$.

If there exist $x_{0}, y_{0} \in X$ such that

$$
x_{0} \preceq F\left(x_{0}, y_{0}\right) \text { and } F\left(y_{0}, x_{0}\right) \preceq y_{0},
$$

then $F$ has a coupled fixed point.

\footnotetext{
*Corresponding author e-mail: habib.yazidi@gmail.com
} 
Inspired by Definition 11, Lakshmikantham and Ćirić in [8] introduced the concept of a $g$-mixed monotone mapping.

Definition 12(see Lakshmikantham and Ćirić [8]). Let $(X, \preceq)$ be a partially ordered set, $F: X \times X \rightarrow X$ and $g: X \rightarrow X$. Then the map $F$ is said to have mixed g-monotone property if $F(x, y)$ is monotone $g$-non-decreasing in $x$ and is monotone g-non-increasing in $y$, that is,

$$
g x_{1} \preceq g x_{2} \text { implies } F\left(x_{1}, y\right) \preceq F\left(x_{2}, y\right) \text { for all } y \in X
$$

and

$$
g y_{1} \preceq g y_{2} \text { implies } F\left(x, y_{2}\right) \preceq F\left(x, y_{1}\right) \text { for all } x \in X \text {. }
$$

Definition 13(Lakshmikantham and Ćirić [8]). Let $X$ be a non-empty set, and let $F: X \times X \rightarrow X, g: X \rightarrow X$ be given mappings. An element $(x, y) \in X \times X$ is called a coupled common fixed point of the mappings $F$ and $g$ if $F(x, y)=$ $g x=x$ and $F(y, x)=g y=y$.

An element $(x, y) \in X \times X$ is called a coupled coincidence point of the mappings $F$ and $g$ if $F(x, y)=g x$ and $F(y, x)=$ gy.

Definition 14(Lakshmikantham and Ćirić [8]). Let X be a non-empty set. Then we say that the mappings $F: X \times X \rightarrow$ $X$ and $g: X \rightarrow X$ are commutative if for all $x, y \in X$

$$
g(F(x, y))=F(g x, g y) .
$$

The main result of Lakshmikantham and Ćirić [8] is the following.

Theorem 12(Lakshmikantham and Ćirić [8]). Let $(X, \preceq)$ be a partially ordered set and suppose there is a metric $d$ on $X$ such that $(X, d)$ is a complete metric space. Assume there is a function $\phi:[0,+\infty) \rightarrow[0,+\infty)$ with $\phi(t)<t$ and $\lim _{r \rightarrow t^{+}} \phi(r)<t$ for each $t>0$ and also suppose $F$ : $X \times X \rightarrow X$ and $g: X \rightarrow X$ are such that $F$ has the mixed $g$-monotone property and

$d(F(x, y), F(u, v)) \leq \phi\left(\frac{d(g x, g u)+d(g y, g v)}{2}\right)$

for all $x, y, u, v \in X$ with $g x \preceq g u$ and $g v \preceq g y$. Assume that $F(X \times X) \subseteq g(X), g$ is continuous and commutes with $F$ and also suppose either $F$ is continuous or $X$ has the following properties:

(i)if a non-decreasing sequence $x_{n} \rightarrow x$, then $x_{n} \preceq x$ for all $n$,

(ii)if a non-increasing sequence $x_{n} \rightarrow x$, then $x \preceq x_{n}$ for all $n$.

If there exist $x_{0}, y_{0} \in X$ such that $g x_{0} \preceq F\left(x_{0}, y_{0}\right)$ and $F\left(y_{0}, x_{0}\right) \preceq g y_{0}$ then there exist $x, y \in X$ such that $g x=F(x, y)$ and $g y=F(y, x)$, that is, $F$ and $g$ have a coupled coincidence point.
Recently, Harjani, López and Sadarangani [7] established coupled fixed point theorems for a mixed monotone operator satisfying contraction involving altering distance functions in a complete partially ordered metric space.

Denote by $\mathscr{F}$ the set of functions $\varphi:[0,+\infty) \rightarrow[0,+\infty)$ satisfying the following properties:

(a) $\varphi$ is continuous and non-decreasing,

(b) $\varphi(t)=0$ if and only if $t=0$.

The functions $\varphi \in \mathscr{F}$ satisfying these properties are called altering distance functions.

Theorem 13(Harjani, López and Sadarangani [7]). Let $(X, \preceq)$ be a partially ordered set and $d$ be a metric on $X$ such that $(X, d)$ is a complete metric space. Let $F: X \times X \rightarrow X$ be a mapping having the mixed monotone property on $X$ and satisfying

$$
\begin{aligned}
\varphi(d(F(x, y), F(u, v)) & \leq \varphi(\max \{d(x, u), d(y, v)\}) \\
& -\Phi(\max \{d(x, u), d(y, v)\})
\end{aligned}
$$

for all $x, y, u, v \in X$ with $u \preceq x$ and $y \preceq v$, where $\varphi, \psi \in$ $\mathscr{F}$. Suppose either $F$ is continuous or $\bar{X}$ has the following properties:

(i)if a non-decreasing sequence $x_{n} \rightarrow x$, then $x_{n} \preceq x$ for all $n$,

(ii)if a non-increasing sequence $x_{n} \rightarrow x$, then $x \preceq x_{n}$ for all $n$.

If there exist $x_{0}, y_{0} \in X$ such that $x_{0} \preceq F\left(x_{0}, y_{0}\right)$ and $F\left(y_{0}, x_{0}\right) \preceq y_{0}$ then $F$ has a coupled fixed point.

In this paper, we introduce the concept of mixed $(G, S)$-monotone mappings and prove coupled coincidence point theorems for such mappings satisfying a nonlinear contraction involving altering distance functions. Presented theorems extend, improve and generalize the results of Harjani, López and Sadarangani [7]. We end this paper by the study of the existence of solutions to a system of nonlinear integral equations.

\section{Main Results}

First, we introduce the concept of mixed $(G, S)$-monotone property.

Definition 21Let $X$ be a non-empty set endowed with a partial order $\preceq$. Consider the mappings $F: X \times X \rightarrow X$ and $G, S: X \rightarrow X$. We say that $F$ has the mixed $(G, S)$-monotone property on $X$ if for all $x, y \in X$,

$x_{1}, x_{2} \in X, \quad G\left(x_{1}\right) \preceq S\left(x_{2}\right) \Rightarrow F\left(x_{1}, y\right) \preceq F\left(x_{2}, y\right)$,

$x_{1}, x_{2} \in X, \quad G\left(x_{1}\right) \succeq S\left(x_{2}\right) \Rightarrow F\left(x_{1}, y\right) \succeq F\left(x_{2}, y\right)$,

$y_{1}, y_{2} \in X, \quad G\left(y_{1}\right) \preceq S\left(y_{2}\right) \Rightarrow F\left(x, y_{1}\right) \succeq F\left(x, y_{2}\right)$,

$y_{1}, y_{2} \in X, \quad G\left(y_{1}\right) \succeq S\left(y_{2}\right) \Rightarrow F\left(x, y_{1}\right) \preceq F\left(x, y_{2}\right)$.

Remark IIf we take $G=S$, then $F$ has the mixed $(G, S)$-monotone property implies that $F$ has the mixed $G$-monotone property. 
Now, we state and prove our first result.

Theorem 21 Let $(X, \preceq)$ be a partially ordered set and suppose that there exists a metric $d$ on $X$ such that $(X, d)$ is a complete metric space. Let $G, S: X \rightarrow X$ and $F: X \times X \rightarrow X$ be a mapping having the mixed $(G, S)$-monotone property on $X$. Suppose that

$$
\begin{aligned}
\varphi(d(F(x, y), F(u, v))) & \leq \varphi(\max \{d(G x, S u), d(G y, S v)\}) \\
& -\phi(\max \{d(G x, S u), d(G y, S v)\}),
\end{aligned}
$$

for all $x, y, u, v \in X$ with $G(x) \preceq S(u)$ or $G(x) \succeq S(u)$ and $S(y) \succeq G(v)$ or $S(y) \preceq G(v)$, where $\varphi, \phi \in \mathscr{F}$. Assume that $F(X \times X) \subseteq G(X) \cap S(X)$ and assume also that $G, S$ and $F$ satisfy the following hypotheses:

(I)F, $G$ and $S$ are continuous,

(II)F commutes respectively with $G$ and $S$.

If there exist $x_{0}, y_{0}, x_{1}$ and $y_{1}$ such that

$$
\left\{\begin{array}{l}
G\left(x_{0}\right) \preceq S\left(x_{1}\right) \preceq F\left(x_{0}, y_{0}\right) \\
G\left(y_{0}\right) \succeq S\left(y_{1}\right) \succeq F\left(y_{0}, x_{0}\right)
\end{array}\right.
$$

then there exist $x, y \in X$ such that

$$
G(x)=S(x)=F(x, y) \quad \text { and } \quad G(y)=S(y)=F(y, x),
$$

that is, G,S and $F$ have a coupled coincidence point $(x, y) \in X \times X$.

Proof. Let $x_{0}, y_{0}, x_{1}, y_{1} \in X$ such that

$G\left(x_{0}\right) \preceq S\left(x_{1}\right) \preceq F\left(x_{0}, y_{0}\right)$ and $G\left(y_{0}\right) \succeq S\left(y_{1}\right) \succeq F\left(y_{0}, x_{0}\right)$.

Since $F(X \times X) \subseteq G(X) \cap S(X)$, we can choose $x_{2}, y_{2}, x_{3}, y_{3} \in X$ such that

$$
\left\{\begin{array}{l}
G\left(x_{2}\right)=F\left(x_{0}, y_{0}\right) \\
G\left(y_{2}\right)=F\left(y_{0}, x_{0}\right)
\end{array}\right.
$$

and

$$
\left\{\begin{array}{l}
S\left(x_{3}\right)=F\left(x_{1}, y_{1}\right) \\
S\left(y_{3}\right)=F\left(y_{1}, x_{1}\right)
\end{array} .\right.
$$

Continuing this process we can construct sequences $\left\{x_{n}\right\}$ and $\left\{y_{n}\right\}$ in $X$ such that

$$
\left\{\begin{array}{l}
G\left(x_{2 n+2}\right)=F\left(x_{2 n}, y_{2 n}\right) \\
G\left(y_{2 n+2}\right)=F\left(y_{2 n}, x_{2 n}\right)
\end{array} ;\left\{\begin{array}{l}
S\left(x_{2 n+3}\right)=F\left(x_{2 n+1}, y_{2 n+1}\right) \\
S\left(y_{2 n+3}\right)=F\left(y_{2 n+1}, x_{2 n+1}\right)
\end{array}\right.\right.
$$

for all $n \geq 0$.

We shall show that for all $n \geq 0$,

$$
G\left(x_{2 n}\right) \preceq S\left(x_{2 n+1}\right) \preceq G\left(x_{2 n+2}\right)
$$

and

$$
G\left(y_{2 n}\right) \succeq S\left(y_{2 n+1}\right) \succeq G\left(y_{2 n+2}\right) .
$$

As $G\left(x_{0}\right) \preceq S\left(x_{1}\right) \preceq F\left(x_{0}, y_{0}\right)=G\left(x_{2}\right)$ and $G\left(y_{0}\right) \succeq S\left(y_{1}\right) \succeq F\left(y_{0}, x_{0}\right)=G\left(y_{2}\right)$, our claim is satisfied for $n=0$.
Suppose that (3) and (4) hold for some fixed $n>0$. Since $G\left(x_{2 n}\right) \preceq \quad S\left(x_{2 n+1}\right) \preceq G\left(x_{2 n+2}\right) \quad$ and $G\left(y_{2 n}\right) \succeq S\left(y_{2 n+1}\right) \succeq G\left(y_{2 n+2}\right)$, and as $F$ has the mixed $(G, S)$-monotone property, we have

$$
\begin{aligned}
G\left(x_{2 n+2}\right)=F\left(x_{2 n}, y_{2 n}\right) & \preceq F\left(x_{2 n+1}, y_{2 n}\right) \\
& \preceq F\left(x_{2 n+1}, y_{2 n+1}\right) \preceq F\left(x_{2 n+2}, y_{2 n+1}\right) \\
& \preceq F\left(x_{2 n+2}, y_{2 n+2}\right),
\end{aligned}
$$

then

$$
G\left(x_{2 n+2}\right) \preceq S\left(x_{2 n+3}\right) \preceq G\left(x_{2 n+4}\right) .
$$

On the other hand,

$$
\begin{aligned}
G\left(y_{2 n+2}\right)=F\left(y_{2 n}, x_{2 n}\right) & \succeq F\left(y_{2 n+1}, x_{2 n}\right) \\
& \succeq F\left(y_{2 n+1}, x_{2 n+1}\right) \succeq F\left(y_{2 n+2}, x_{2 n+1}\right) \\
& \succeq F\left(y_{2 n+2}, x_{2 n+2}\right),
\end{aligned}
$$

then

$$
G\left(y_{2 n+2}\right) \succeq S\left(y_{2 n+3}\right) \succeq G\left(y_{2 n+4}\right) .
$$

Thus by induction, we proved that (3) and (4) hold for all $n \geq 0$.

We complete the proof in the following steps:

Step 1: We will prove that

$$
\begin{aligned}
& \lim _{n \rightarrow+\infty} d\left(F\left(x_{n}, y_{n}\right), F\left(x_{n+1}, y_{n+1}\right)\right)= \\
& \lim _{n \rightarrow+\infty} d\left(F\left(y_{n}, x_{n}\right), F\left(y_{n+1}, x_{n+1}\right)\right)=0 .
\end{aligned}
$$

From (3), (4) and (1), we have

$$
\begin{aligned}
& \varphi\left(d\left(F\left(x_{2 n}, y_{2 n}\right), F\left(x_{2 n+1}, y_{2 n+1}\right)\right)\right) \\
& \leq \varphi\left(\max \left\{d\left(G x_{2 n}, S x_{2 n+1}\right), d\left(G y_{2 n}, S y_{2 n+1}\right)\right\}\right) \\
& -\phi\left(\max \left\{d\left(G x_{2 n}, S x_{2 n+1}\right), d\left(G y_{2 n}, S y_{2 n+1}\right)\right\}\right) \\
& \leq \varphi\left(\max \left\{d\left(G x_{2 n}, S x_{2 n+1}\right), d\left(G y_{2 n}, S y_{2 n+1}\right)\right\}\right) .
\end{aligned}
$$

Since $\varphi$ is a non-decreasing function, we get that

$$
d\left(F\left(x_{2 n}, y_{2 n}\right), F\left(x_{2 n+1}, y_{2 n+1}\right)\right) \leq
$$

$\max \left\{d\left(G x_{2 n}, S x_{2 n+1}\right), d\left(G y_{2 n}, S y_{2 n+1}\right)\right\}$.

Therefore

$d\left(G x_{2 n+2}, S x_{2 n+3}\right) \leq \max \left\{d\left(G x_{2 n}, S x_{2 n+1}\right), d\left(G y_{2 n}, S y_{2 n+1}\right)\right\}$.

Again, using (3), (4) and (1), we have

$$
\begin{aligned}
& \varphi\left(d\left(F\left(y_{2 n}, x_{2 n}\right), F\left(y_{2 n+1}, x_{2 n+1}\right)\right)\right) \\
& \leq \varphi\left(\max \left\{d\left(G y_{2 n}, S y_{2 n+1}\right), d\left(G x_{2 n}, S x_{2 n+1}\right)\right\}\right) \\
& -\phi\left(\max \left\{d\left(G y_{2 n}, S y_{2 n+1}\right), d\left(G x_{2 n}, S x_{2 n+1}\right)\right\}\right) \\
& \leq \varphi\left(\max \left\{d\left(G y_{2 n}, S y_{2 n+1}\right), d\left(G x_{2 n}, S x_{2 n+1}\right)\right\}\right) .
\end{aligned}
$$

Since $\varphi$ is non-decreasing, we have

$$
\begin{aligned}
& d\left(F\left(y_{2 n}, x_{2 n}\right), F\left(y_{2 n+1}, x_{2 n+1}\right)\right) \leq \\
& \quad \max \left\{d\left(G y_{2 n}, S y_{2 n+1}\right), d\left(G x_{2 n}, S x_{2 n+1}\right\} .\right.
\end{aligned}
$$


Therefore

$$
\begin{array}{r}
d\left(G y_{2 n+2}, S y_{2 n+3}\right) \leq \\
\max \left\{d\left(G y_{2 n}, S y_{2 n+1}\right), d\left(G x_{2 n}, S x_{2 n+1}\right)\right\} .
\end{array}
$$

Combining (7) and (9), we obtain

$$
\begin{aligned}
\max \left\{d\left(G x_{2 n+2}, S x_{2 n+3}\right), d\left(G y_{2 n+2}, S y_{2 n+3}\right)\right\} \\
\leq \max \left\{d\left(G x_{2 n}, S x_{2 n+1}\right), d\left(G y_{2 n}, S y_{2 n+1}\right)\right\} .
\end{aligned}
$$

Then $\left\{\max \left\{d\left(G x_{2 n}, S x_{2 n+1}\right), d\left(G y_{2 n}, S y_{2 n+1}\right)\right\}\right\}$ is a positive non-increasing sequence. Hence there exists $r \geq 0$ such that

$$
\lim _{n \rightarrow+\infty} \max \left\{d\left(G x_{2 n}, S x_{2 n+1}\right), d\left(G y_{2 n}, S y_{2 n+1}\right)\right\}=r .
$$

Combining (6) and (8), we obtain

$$
\begin{aligned}
& \max \left\{\varphi\left(d\left(G x_{2 n+2}, S x_{2 n+3}\right)\right), \varphi\left(d\left(G y_{2 n+2}, S y_{2 n+3}\right)\right)\right\} \\
& \leq \varphi\left(\max \left\{d\left(G x_{2 n}, S x_{2 n+1}\right), d\left(G y_{2 n}, S y_{2 n+1}\right)\right\}\right) \\
& -\phi\left(\max \left\{d\left(G x_{2 n}, S x_{2 n+1}\right), d\left(G y_{2 n}, S y_{2 n+1}\right)\right\}\right)
\end{aligned}
$$

Since $\varphi$ is non-decreasing, we get

$$
\begin{aligned}
& \varphi\left(\max \left\{d\left(G x_{2 n+2}, S x_{2 n+3}\right), d\left(G y_{2 n+2}, S y_{2 n+3}\right)\right\}\right) \\
& \leq \varphi\left(\max \left\{d\left(G x_{2 n}, S x_{2 n+1}\right), d\left(G y_{2 n}, S y_{2 n+1}\right)\right\}\right) \\
& -\phi\left(\max \left\{d\left(G x_{2 n}, S x_{2 n+1}\right), d\left(G y_{2 n}, S y_{2 n+1}\right)\right\}\right) .
\end{aligned}
$$

Letting $n \rightarrow+\infty$ in the above inequality, we get

$$
\varphi(r) \leq \varphi(r)-\phi(r),
$$

which implies that $\phi(r)=0$ and then, since $\phi$ is an altering distance function, $r=0$. Consequently

$$
\begin{aligned}
\lim _{n \rightarrow+\infty} \max \{d( & \left(F\left(x_{2 n}, y_{2 n}\right), F\left(x_{2 n+1}, y_{2 n+1}\right)\right), \\
d( & \left.\left.\left(y_{2 n}, x_{2 n}\right), F\left(y_{2 n+1}, x_{2 n+1}\right)\right)\right\}=0 .
\end{aligned}
$$

By the same way, we obtain

$$
\begin{gathered}
\lim _{n \rightarrow+\infty} \max \left\{d\left(F\left(x_{2 n+1}, y_{2 n+1}\right), F\left(x_{2 n+2}, y_{2 n+2}\right)\right),\right. \\
\left.d\left(F\left(y_{2 n+1}, x_{2 n+1}\right), F\left(y_{2 n+2}, x_{2 n+2}\right)\right)\right\}=0 .
\end{gathered}
$$

Finally, (10) and (11) give the desired result, that is, (5) holds.

Step 2: We will prove that $F\left(x_{n}, y_{n}\right)$ and $F\left(y_{n}, x_{n}\right)$ are Cauchy sequences.

From (5), it is sufficient to show that $F\left(x_{2 n}, y_{2 n}\right)$ and $F\left(y_{2 n}, x_{2 n}\right)$ are Cauchy sequences.

We proceed by negation and suppose that at least one of the sequences $F\left(x_{2 n}, y_{2 n}\right)$ or $F\left(y_{2 n}, x_{2 n}\right)$ is not a Cauchy sequence.

This implies that $d\left(F\left(x_{2 n}, y_{2 n}\right), F\left(x_{2 m}, y_{2 m}\right)\right) \nrightarrow 0$ or
$d\left(F\left(y_{2 n}, x_{2 n}\right), F\left(y_{2 m}, x_{2 m}\right)\right) \nrightarrow 0$ as $n, m \rightarrow+\infty$.

Consequently

$$
\begin{aligned}
& \max \left\{d\left(F\left(x_{2 n}, y_{2 n}\right), F\left(x_{2 m}, y_{2 m}\right)\right),\right. \\
& \left.d\left(F\left(y_{2 n}, x_{2 n}\right), F\left(y_{2 m}, x_{2 m}\right)\right)\right\} \nrightarrow 0, \text { as } n, m \rightarrow+\infty
\end{aligned}
$$

Then there exists $\varepsilon>0$ for which we can find two subsequences of positive integers $\{m(i)\}$ and $\{n(i)\}$ such that $n(i)$ is the smallest index for which $n(i)>m(i)>i$,

$$
\begin{aligned}
& \max \left\{d\left(F\left(x_{2 m(i)}, y_{2 m(i)}\right), F\left(x_{2 n(i)}, y_{2 n(i)}\right)\right),\right. \\
& \left.d\left(F\left(y_{2 m(i)}, x_{2 m(i)}\right), F\left(y_{2 n(i)}, x_{2 n(i)}\right)\right)\right\} \geq \varepsilon .
\end{aligned}
$$

This means that

$$
\begin{aligned}
& \max \left\{d\left(F\left(x_{2 m(i)}, y_{2 m(i)}\right), F\left(x_{2 n(i)-2}, y_{2 n(i)-2}\right)\right),\right. \\
& \left.d\left(F\left(y_{2 m(i)}, x_{2 m(i)}\right), F\left(y_{2 n(i)-2}, x_{2 n(i)-2}\right)\right)\right\}<\varepsilon .
\end{aligned}
$$

From (12), (13) and using the triangular inequality, we get

$$
\begin{array}{r}
\varepsilon \leq \max \left\{d\left(F\left(x_{2 m(i)}, y_{2 m(i)}\right), F\left(x_{2 n(i)}, y_{2 n(i)}\right)\right),\right. \\
\left.d\left(F\left(y_{2 m(i)}, x_{2 m(i)}\right), F\left(y_{2 n(i)}, x_{2 n(i)}\right)\right)\right\} \\
\leq \max \left\{d\left(F\left(x_{2 m(i)}, y_{2 m(i)}\right), F\left(x_{2 n(i)-2}, y_{2 n(i)-2}\right)\right),\right. \\
\left.d\left(F\left(y_{2 m(i)}, x_{2 m(i)}\right), F\left(y_{2 n(i)-2}, x_{2 n(i)-2}\right)\right)\right\}
\end{array}
$$

$$
+\max \left\{d\left(F\left(x_{2 n(i)-2}, y_{2 n(i)-2}\right), F\left(x_{2 n(i)-1}, y_{2 n(i)-1}\right)\right),\right.
$$

$$
\left.d\left(F\left(y_{2 n(i)-2}, x_{2 n(i)-2}\right), F\left(y_{2 n(i)-1}, x_{2 n(i)-1}\right)\right)\right\}
$$$$
+\max \left\{d\left(F\left(x_{2 n(i)-1}, y_{2 n(i)-1}\right), F\left(x_{2 n(i)}, y_{2 n(i)}\right)\right),\right.
$$

$$
\left.d\left(\left(F\left(y_{2 n(i)-1}, x_{2 n(i)-1}\right), F\left(y_{2 n(i)}, x_{2 n(i)}\right)\right)\right)\right\}
$$

$$
\begin{array}{r}
<\varepsilon+\max \left\{d\left(F\left(x_{2 n(i)-2}, y_{2 n(i)-2}\right), F\left(x_{2 n(i)-1}, y_{2 n(i)-1}\right)\right),\right. \\
\left.d\left(F\left(y_{2 n(i)-2}, x_{2 n(i)-2}\right), F\left(y_{2 n(i)-1}, x_{2 n(i)-1}\right)\right)\right\} \\
+\max \left\{d\left(F\left(x_{2 n(i)-1}, y_{2 n(i)-1}\right), F\left(x_{2 n(i)}, y_{2 n(i)}\right)\right),\right. \\
\left.d\left(F\left(y_{2 n(i)-1}, x_{2 n(i)-1}\right), F\left(y_{2 n(i)}, x_{2 n(i)}\right)\right)\right\} .
\end{array}
$$

Letting $i \rightarrow+\infty$ in above inequality and using (5), we obtain that

$$
\begin{aligned}
& \lim _{i \rightarrow+\infty} \max \left\{d\left(F\left(x_{2 m(i)}, y_{2 m(i)}\right), F\left(x_{2 n(i)}, y_{2 n(i)}\right)\right),\right. \\
& \left.d\left(F\left(y_{2 m(i)}, x_{2 m(i)}\right), F\left(y_{2 n(i)}, x_{2 n(i)}\right)\right)\right\}=\varepsilon .
\end{aligned}
$$


Also, we have

$$
\begin{array}{r}
\varepsilon \leq \max \left\{d\left(F\left(x_{2 m(i)}, y_{2 m(i)}\right), F\left(x_{2 n(i)}, y_{2 n(i)}\right)\right),\right. \\
\left.d\left(F\left(y_{2 m(i)}, x_{2 m(i)}\right), F\left(y_{2 n(i)}, x_{2 n(i)}\right)\right)\right\} \\
\leq \max \left\{d\left(F\left(x_{2 m(i)}, y_{2 m(i)}\right), F\left(x_{2 n(i)-1}, y_{2 n(i)-1}\right)\right),\right. \\
\left.d\left(F\left(y_{2 m(i)}, x_{2 m(i)}\right), F\left(y_{2 n(i)-1}, x_{2 n(i)-1}\right)\right)\right\} \\
+\max \left\{d\left(F\left(x_{2 n(i)-1}, y_{2 n(i)-1}\right), F\left(x_{2 n(i)}, y_{2 n(i)}\right)\right),\right. \\
\left.d\left(F\left(y_{2 n(i)-1}, x_{2 n(i)-1}\right), F\left(y_{2 n(i)}, x_{2 n(i)}\right)\right)\right\} \\
\leq \max \left\{d\left(F\left(x_{2 m(i)}, y_{2 m(i)}\right), F\left(x_{2 n(i)}, y_{2 n(i)}\right)\right),\right. \\
\left.d\left(F\left(y_{2 m(i)}, x_{2 m(i)}\right), F\left(y_{2 n(i)}, x_{2 n(i)}\right)\right)\right\} \\
+\max \left\{d\left(F\left(x_{2 n(i)}, y_{2 n(i)}\right), F\left(x_{2 n(i)-1}, y_{2 n(i)-1}\right)\right),\right. \\
\left.d\left(F\left(y_{2 n(i)}, x_{2 n(i)}\right), F\left(y_{2 n(i)-1}, x_{2 n(i)-1}\right)\right)\right\} \\
+\max \left\{d\left(F\left(x_{2 n(i)-1}, y_{2 n(i)-1}\right), F\left(x_{2 n(i)}, y_{2 n(i)}\right)\right),\right. \\
\left.d\left(F\left(y_{2 m(i)}, x_{2 m(i)}\right), F\left(y_{2 n(i)}, x_{2 n(i)}\right)\right)\right\} .
\end{array}
$$

Using (5), (14) and letting $i \rightarrow+\infty$ in the above inequality, we obtain

$$
\begin{aligned}
& \lim _{i \rightarrow+\infty} \max \left\{d\left(F\left(x_{2 m(i)}, y_{2 m(i)}\right), F\left(x_{2 n(i)-1}, y_{2 n(i)-1}\right)\right),\right. \\
& \left.d\left(F\left(y_{2 m(i)}, x_{2 m(i)}\right), F\left(y_{2 n(i)-1}, x_{2 n(i)-1}\right)\right)\right\}=\varepsilon .
\end{aligned}
$$

On other hand, we have

$$
\begin{array}{r}
\max \left\{d\left(F\left(x_{2 m(i)}, y_{2 m(i)}\right), F\left(x_{2 n(i)}, y_{2 n(i)}\right)\right),\right. \\
\left.d\left(F\left(y_{2 m(i)}, x_{2 m(i)}\right), F\left(y_{2 n(i)}, x_{2 n(i)}\right)\right)\right\} \\
\leq \max \left\{d\left(F\left(x_{2 m(i)}, y_{2 m(i)}\right), F\left(x_{2 m(i)+1}, y_{2 m(i)+1}\right)\right),\right. \\
\left.d\left(F\left(y_{2 m(i)}, x_{2 m(i)}\right), F\left(y_{2 m(i)+1}, x_{2 m(i)+1}\right)\right)\right\} \\
+\max \left\{d\left(F\left(x_{2 m(i)+1}, y_{2 m(i)+1}\right), F\left(x_{2 m(i)+2}, y_{2 m(i)+2}\right)\right),\right. \\
\left.d\left(F\left(y_{2 m(i)+1}, x_{2 m(i)+1}\right), F\left(y_{2 m(i)+2}, x_{2 m(i)+2}\right)\right)\right\} \\
+\max \left\{d\left(F\left(x_{2 m(i)+2}, y_{2 n(i)+1}\right), F\left(x_{2 n(i)+1}, y_{2 n(i)+1}\right)\right),\right. \\
\left.d\left(F\left(y_{2 m(i)+2}, x_{2 m(i)+2}\right), F\left(y_{2 n(i)+1}, x_{2 n(i)+1}\right)\right)\right\} \\
+\max \left\{d\left(F\left(x_{2 n(i)+1}, y_{2 n(i)+1}\right), F\left(x_{2 n(i)}, y_{2 n(i)}\right)\right),\right. \\
\left.d\left(F\left(y_{2 n(i)+1}, x_{2 n(i)+1}\right), F\left(y_{2 n(i)}, x_{2 n(i)}\right)\right)\right\} .
\end{array}
$$

Since $\varphi$ is a continuous non-decreasing function, using (5) in the above inequality, we get taking the upper limit

$$
\begin{gathered}
\varphi(\varepsilon) \leq \quad \varphi\left(\operatorname { l i m s u p } _ { i \rightarrow + \infty } \operatorname { m a x } \left\{d \left(F\left(x_{2 m(i)+2}, y_{2 m(i)+2}\right),\right.\right.\right. \\
\left.F\left(x_{2 n(i)+1}, y_{2 n(i)+1}\right)\right), d\left(F\left(y_{2 m(i)+2}, x_{2 m(i)+2}\right),\right. \\
\left.\left.F\left(y_{2 n(i)+1}, x_{2 n(i)+1}\right)\right\}\right) .
\end{gathered}
$$

Using the contractive condition (1), on one hand we have

$$
\begin{aligned}
& \varphi\left(d\left(F\left(x_{2 m(i)+2}, y_{2 m(i)+2}\right), F\left(x_{2 n(i)+1}, y_{2 n(i)+1}\right)\right)\right. \\
& \leq \varphi\left(\max \left\{d\left(G x_{2 m(i)+2}, S x_{2 n(i)+1}\right), d\left(G y_{2 m(i)+2}, S y_{2 n(i)+1}\right)\right\}\right) \\
& -\phi\left(\max \left\{d\left(G x_{2 m(i)+2}, S x_{2 n(i)+1}\right), d\left(G y_{2 m(i)+2}, S y_{2 n(i)+1}\right)\right\}\right) \\
& =\varphi\left(\operatorname { m a x } \left\{d\left(F\left(x_{2 m(i)}, y_{2 m(i)}\right), F\left(x_{2 n(i)-1}, y_{2 n(i)-1}\right)\right),\right.\right. \\
& \left.\left.d\left(F\left(y_{2 m(i)}, x_{2 m(i)}\right), F\left(y_{2 n(i)-1}, x_{2 n(i)-1}\right)\right)\right\}\right) \\
& -\phi\left(\operatorname { m a x } \left\{d\left(F\left(x_{2 m(i)}, y_{2 m(i)}\right), F\left(x_{2 n(i)-1}, y_{2 n(i)-1}\right)\right),\right.\right. \\
& \left.\left.d\left(F\left(y_{2 m(i)}, x_{2 m(i)}\right), F\left(y_{2 n(i)-1}, x_{2 n(i)-1}\right)\right)\right\}\right) .
\end{aligned}
$$

On the other hand, we have

$$
\begin{aligned}
& \varphi\left(d\left(F\left(y_{2 m(i)+2}, x_{2 m(i)+2}\right), F\left(y_{2 n(i)+1}, x_{2 n(i)+1}\right)\right)\right) \\
& \leq \varphi\left(\operatorname { m a x } \left\{d\left(G y_{2 m(i)+2}, S y_{2 n(i)+1}\right),\right.\right. \\
& \left.\left.d\left(G x_{2 m(i)+2}, S x_{2 n(i)+1}\right)\right\}\right) \\
& -\phi\left(\operatorname { m a x } \left\{d\left(G y_{2 m(i)+2}, S y_{2 n(i)+1}\right),\right.\right. \\
& \left.\left.d\left(G x_{2 m(i)+2}, S x_{2 n(i)+1}\right)\right\}\right) \\
& =\varphi\left(\operatorname { m a x } \left\{d\left(F\left(y_{2 m(i)}, x_{2 m(i)}\right), F\left(y_{2 n(i)-1}, x_{2 n(i)-1}\right)\right),\right.\right. \\
& \left.\left.d\left(F\left(x_{2 m(i)}, y_{2 m(i)}\right), F\left(x_{2 n(i)-1}, y_{2 n(i)-1}\right)\right)\right\}\right) \\
& -\phi\left(\operatorname { m a x } \left\{d\left(F\left(y_{2 m(i)}, x_{2 m(i)}\right)\right), F\left(y_{2 n(i)-1}, x_{2 n(i)-1}\right),\right.\right. \\
& \left.\left.d\left(F\left(x_{2 m(i)}, y_{2 m(i)}\right)\right), F\left(x_{2 n(i)-1}, y_{2 n(i)-1}\right)\right\}\right) .
\end{aligned}
$$

\section{Therefore}

$$
\begin{array}{r}
\varphi\left(\operatorname { m a x } \left\{d\left(F\left(x_{2 m(i)+2}, y_{2 m(i)+2}\right), F\left(x_{2 n(i)+1}, y_{2 n(i)+1}\right)\right),\right.\right. \\
\left.\left.d\left(F\left(y_{2 m(i)+2}, x_{2 m(i)+2}\right), F\left(y_{2 n(i)+1}, x_{2 n(i)+1}\right)\right)\right\}\right) \\
\leq \max \left\{\varphi \left(d\left(F\left(x_{2 m(i)+2}, y_{2 m(i)+2}\right), F\left(x_{2 n(i)+1}, y_{2 n(i)+1}\right)\right),\right.\right. \\
\varphi\left(d\left(F\left(y_{2 m(i)+2}, x_{2 m(i)+2}\right), F\left(y_{2 n(i)+1}, x_{2 n(i)+1}\right)\right)\right\} \\
\leq \varphi\left(\operatorname { m a x } \left\{d\left(F\left(x_{2 m(i)}, y_{2 m(i)}\right), F\left(x_{2 n(i)-1}, y_{2 n(i)-1}\right)\right),\right.\right. \\
\left.d\left(F\left(y_{2 m(i)}, x_{2 m(i)}\right), F\left(y_{2 n(i)-1}, x_{2 n(i)-1}\right)\right)\right\} \\
-\phi\left(\operatorname { m a x } \left\{d\left(F\left(x_{2 m(i)}, y_{2 m(i)}\right), F\left(x_{2 n(i)-1}, y_{2 n(i)-1}\right)\right),\right.\right. \\
\left.\left.d\left(F\left(y_{2 m(i)}, x_{2 m(i)}\right), F\left(y_{2 n(i)-1}, x_{2 n(i)-1}\right)\right)\right\}\right) .
\end{array}
$$

Finally, taking the limsup as $i \rightarrow+\infty$ in (17), using (15), (16) and the continuity of $\varphi$ and $\phi$, we get

$$
\varphi(\varepsilon) \leq \varphi(\varepsilon)-\phi(\varepsilon)
$$

which implies that $\phi(\varepsilon)=0$, that is, $\varepsilon=0$, a contradiction. Thus $\left\{F\left(x_{2 n}, y_{2 n}\right)\right\}$ and $\left\{F\left(y_{2 n}, x_{2 n}\right)\right\}$ are Cauchy sequences in $X$, which give us that $\left\{F\left(x_{n}, y_{n}\right)\right\}$ and $\left\{F\left(y_{n}, x_{n}\right)\right\}$ are also Cauchy sequences.

Step 3: Existence of a coupled coincidence point. Since $\left\{F\left(x_{n}, y_{n}\right)\right\}$ and $\left\{F\left(y_{n}, x_{n}\right)\right\}$ are Cauchy sequences in 
the complete metric space $(X, d)$, there exist $\alpha, \alpha^{\prime} \in X$ such that

$$
\lim _{n \rightarrow+\infty} F\left(x_{n}, y_{n}\right)=\alpha \text { and } \lim _{n \rightarrow+\infty} F\left(y_{n}, x_{n}\right)=\alpha^{\prime} .
$$

Therefore, $\lim _{n \rightarrow+\infty} G\left(x_{2 n+2}\right)=\alpha, \lim _{n \rightarrow+\infty} G\left(y_{2 n+2}\right)=\alpha^{\prime}$, $\lim _{n \rightarrow+\infty} S\left(x_{2 n+3}\right)=\alpha$ and $\lim _{n \rightarrow+\infty} S\left(y_{2 n+3}\right)=\alpha^{\prime}$.

Using the continuity and the commutativity of $F$ and $G$, we have

$$
\begin{aligned}
G\left(G\left(x_{2 n+2}\right)\right) & =G\left(F\left(x_{2 n}, y_{2 n}\right)\right) \\
& =F\left(G x_{2 n}, G y_{2 n}\right)
\end{aligned}
$$

and

$$
\begin{aligned}
G\left(G\left(y_{2 n+2}\right)\right) & =G\left(F\left(y_{2 n}, x_{2 n}\right)\right) \\
& =F\left(G y_{2 n}, G x_{2 n}\right) .
\end{aligned}
$$

Letting $n \rightarrow+\infty$, we get $G(\alpha)=F\left(\alpha, \alpha^{\prime}\right)$ and $G\left(\alpha^{\prime}\right)=F\left(\alpha^{\prime}, \alpha\right)$.

Using also the continuity and the commutativity of $F$ and $S$, by the same way, we obtain $S(\alpha)=F\left(\alpha, \alpha^{\prime}\right)$ and $S\left(\alpha^{\prime}\right)=F\left(\alpha^{\prime}, \alpha\right)$.

Therefore,

$G(\alpha)=F\left(\alpha, \alpha^{\prime}\right)=S(\alpha) \quad$ and $\quad G\left(\alpha^{\prime}\right)=F\left(\alpha^{\prime}, \alpha\right)=S\left(\alpha^{\prime}\right)$

Thus we proved that $\left(\alpha, \alpha^{\prime}\right)$ is a coupled coincidence point of $G, S$ and $F$.

In the next result, we prove that the previous theorem is still valid if we replace the continuity of $F$ by some conditions.

Theorem 22If we replace the continuity hypothesis of $F$ in Theorem 21 by the following conditions:

(i)if $\left(x_{n}\right)$ is a non-decreasing sequences with $x_{n} \rightarrow x$ then $x_{n} \preceq x$ for each $n \in \mathbb{N}$,

(ii)if $\left(y_{n}\right)$ is a non-increasing sequences with $y_{n} \rightarrow y$ then $y \preceq y_{n}$ for each $n \in \mathbb{N}$,

(iii) $x, y \in X, \quad x \preceq y \Rightarrow G x \preceq S y$,

(iv) $x, y \in X, \quad x \succeq y \Rightarrow G x \succeq S y$.

\section{Then $G, S$ and $F$ have a coupled coincidence point.}

Proof. Following the proof of Theorem 21, we have that $F\left(x_{n}, y_{n}\right)$ and $F\left(y_{n}, x_{n}\right)$ are Cauchy sequences in the complete metric space $(X, d)$, there exist $\alpha, \alpha^{\prime} \in X$ such that

$$
\lim _{n \rightarrow+\infty} F\left(x_{n}, y_{n}\right)=\alpha \text { and } \lim _{n \rightarrow+\infty} F\left(y_{n}, x_{n}\right)=\alpha^{\prime} .
$$

Therefore, $\quad \lim _{n \rightarrow+\infty} F\left(x_{2 n}, y_{2 n}\right)=\alpha \quad$ and $\lim _{n \rightarrow+\infty} F\left(y_{2 n}, x_{2 n}\right) \stackrel{n \rightarrow+\infty}{=} \alpha^{\prime}$. Hence, $\lim _{n \rightarrow+\infty} G\left(x_{2 n+2}\right)=\alpha$, $\lim _{n \rightarrow+\infty} G\left(y_{2 n+2}\right)=\alpha^{\prime}, \quad \lim _{n \rightarrow+\infty} S\left(x_{2 n+3}\right)=\alpha$ and $\lim _{n \rightarrow+\infty} S\left(y_{2 n+3}\right)=\alpha^{\prime}$. Using the commutativity of $\{F, G\}$ and $\{F, S\}$ and the contractive condition (1), it follows from the conditions (iii) and (iv) that

$$
\begin{aligned}
& \varphi\left(d\left(G\left(F\left(x_{2 n}, y_{2 n}\right)\right), S\left(F\left(x_{2 n+1}, y_{2 n+1}\right)\right)\right)\right) \\
& =\varphi\left(d\left(F\left(G x_{2 n}, G y_{2 n}\right), F\left(S x_{2 n+1}, S y_{2 n+1}\right)\right)\right) \\
& \leq \varphi\left(\operatorname { m a x } \left\{d\left(G\left(G x_{2 n}\right), S\left(S x_{2 n+1}\right)\right)\right.\right. \\
& \left.\left.d\left(G\left(G y_{2 n}\right), S\left(S y_{2 n+1}\right)\right)\right\}\right)
\end{aligned}
$$$$
-\phi\left(\max \left\{d\left(G\left(G x_{2 n}\right), S\left(S x_{2 n+1}\right)\right), d\left(G\left(G y_{2 n}\right), S\left(S y_{2 n+1}\right)\right)\right\}\right) .
$$

Similarly, we have

$$
\begin{aligned}
& \varphi\left(d\left(G\left(F\left(y_{2 n}, x_{2 n}\right)\right), S\left(F\left(y_{2 n+1}, x_{2 n+1}\right)\right)\right)\right) \\
& =\varphi\left(d\left(F\left(G y_{2 n}, G x_{2 n}\right), F\left(S y_{2 n+1}, S x_{2 n+1}\right)\right)\right) \\
& \leq \varphi\left(\operatorname { m a x } \left\{d\left(G\left(G y_{2 n}\right), S\left(S y_{2 n+1}\right)\right)\right.\right. \\
& \left.\left.d\left(G\left(G x_{2 n}\right), S\left(S x_{2 n+1}\right)\right)\right\}\right) \\
& -\phi\left(\operatorname { m a x } \left\{d\left(G\left(G y_{2 n}\right), S\left(S y_{2 n+1}\right)\right)\right.\right. \\
& \left.\left.d\left(G\left(G x_{2 n}\right), S\left(S x_{2 n+1}\right)\right)\right\}\right) .
\end{aligned}
$$

Combining (18), (19) and the fact that $\max \{\varphi(a), \varphi(b)\}=$ $\varphi(\max \{a, b\})$ for $a, b \in[0,+\infty)$, from (iii) and (iv), we obtain

$$
\begin{aligned}
& \varphi\left(\operatorname { m a x } \left\{d\left(G\left(F\left(x_{2 n}, y_{2 n}\right)\right), S\left(F\left(x_{2 n+1}, y_{2 n+1}\right)\right)\right),\right.\right. \\
& \left.\left.d\left(G\left(F\left(y_{2 n}, x_{2 n}\right)\right), S\left(F\left(y_{2 n+1}, x_{2 n+1}\right)\right)\right)\right\}\right) \\
& \leq \varphi\left(\operatorname { m a x } \left\{d\left(G\left(G x_{2 n}\right), S\left(S x_{2 n+1}\right)\right),\right.\right. \\
& \left.\left.d\left(G\left(G y_{2 n}\right), S\left(S y_{2 n+1}\right)\right)\right\}\right) \\
& -\phi\left(\operatorname { m a x } \left\{d\left(G\left(G x_{2 n}\right), S\left(S x_{2 n+1}\right)\right),\right.\right. \\
& \left.\left.d\left(G\left(G y_{2 n}\right), S\left(S y_{2 n+1}\right)\right)\right\}\right) .
\end{aligned}
$$

Letting $n \rightarrow+\infty$ in the last expression, using the continuity of $G$ and $S$, we get

$$
\begin{aligned}
& \varphi\left(\max \left\{d(G(\alpha), S(\alpha)), d\left(G\left(\alpha^{\prime}\right), S\left(\alpha^{\prime}\right)\right)\right\}\right) \\
& \leq \varphi\left(\max \left\{d(G(\alpha), S(\alpha)), d\left(G\left(\alpha^{\prime}\right), S\left(\alpha^{\prime}\right)\right)\right\}\right) \\
& -\phi\left(\max \left\{d(G(\alpha), S(\alpha)), d\left(G\left(\alpha^{\prime}\right), S\left(\alpha^{\prime}\right)\right)\right\}\right) .
\end{aligned}
$$

This implies that $\phi\left(\max \left\{d(G(\alpha), S(\alpha)), d\left(G\left(\alpha^{\prime}\right), S\left(\alpha^{\prime}\right)\right)\right\}\right)=0$ and, since $\phi$ is an altering distance function, then

$$
\max \left\{d(G(\alpha), S(\alpha)), d\left(G\left(\alpha^{\prime}\right), S\left(\alpha^{\prime}\right)\right)\right\}=0
$$

Consequently

$G(\alpha)=S(\alpha) \quad$ and $\quad G\left(\alpha^{\prime}\right)=S\left(\alpha^{\prime}\right)$

To finish the proof, we claim that $F\left(\alpha, \alpha^{\prime}\right)=G(\alpha)=S(\alpha)$ and $F\left(\alpha^{\prime}, \alpha\right)=G\left(\alpha^{\prime}\right)=S\left(\alpha^{\prime}\right)$. 
Indeed, using the contractive condition (1), (3) and (4), it follows from (i)-(iv) that

$$
\begin{aligned}
& \varphi\left(d\left(F\left(G x_{2 n}, G y_{2 n}\right), F\left(\alpha, \alpha^{\prime}\right)\right)\right) \\
& \leq \varphi\left(\max \left\{d\left(G\left(G x_{2 n}\right), S(\alpha)\right), d\left(G\left(G y_{2 n}\right), S\left(\alpha^{\prime}\right)\right)\right\}\right) \\
& -\phi\left(\max \left\{d\left(G\left(G x_{2 n}\right), S(\alpha)\right), d\left(G\left(G y_{2 n}\right), S\left(\alpha^{\prime}\right)\right)\right\}\right) \\
& \leq \varphi\left(\max \left\{d\left(G\left(G x_{2 n}\right), S(\alpha)\right), d\left(G\left(G y_{2 n}\right), S\left(\alpha^{\prime}\right)\right)\right\}\right) .
\end{aligned}
$$

Using the fact that $\varphi$ is non-decreasing, we get

$$
\begin{aligned}
& d\left(F\left(G x_{2 n}, G y_{2 n}\right), F\left(\alpha, \alpha^{\prime}\right)\right) \leq \\
& \max \left\{d\left(G\left(G x_{2 n}\right), S(\alpha)\right), d\left(G\left(G y_{2 n}\right), S\left(\alpha^{\prime}\right)\right)\right\} .
\end{aligned}
$$

Similarly, we have

$$
\begin{array}{r}
\varphi\left(d\left(F\left(G y_{2 n}, G x_{2 n}\right), F\left(\alpha^{\prime}, \alpha\right)\right)\right) \\
\leq \varphi\left(\max \left\{d\left(G\left(G y_{2 n}\right), S\left(\alpha^{\prime}\right)\right), d\left(G\left(G x_{2 n}\right), S(\alpha)\right)\right\}\right) \\
-\phi\left(\max \left\{d\left(G\left(G y_{2 n}\right), S\left(\alpha^{\prime}\right)\right), d\left(G\left(G x_{2 n}\right), S(\alpha)\right)\right\}\right. \\
\leq \varphi\left(\max \left\{d\left(G\left(G y_{2 n}\right), S\left(\alpha^{\prime}\right)\right), d\left(G\left(G x_{2 n}\right), S(\alpha)\right)\right\}\right) .
\end{array}
$$

Using the fact that $\varphi$ is non-decreasing, we see that

$$
\begin{aligned}
& d\left(F\left(G y_{2 n}, G x_{2 n}\right), F\left(\alpha^{\prime}, \alpha\right)\right) \leq \\
& \max \left\{d\left(G\left(G y_{2 n}\right), S\left(\alpha^{\prime}\right)\right), d\left(G\left(G x_{2 n}\right), S(\alpha)\right)\right\} .
\end{aligned}
$$

Combining (21) and (22), we get

$$
\begin{aligned}
& \max \left\{d\left(F\left(G x_{2 n}, G y_{2 n}\right), F\left(\alpha, \alpha^{\prime}\right)\right),\right. \\
& \left.d\left(F\left(G y_{2 n}, G x_{2 n}\right), F\left(\alpha^{\prime}, \alpha\right)\right)\right) \\
& \leq \max \left\{d\left(G\left(G x_{2 n}\right), S(\alpha)\right), d\left(G\left(G y_{2 n}\right), S\left(\alpha^{\prime}\right)\right)\right\} .
\end{aligned}
$$

Using the commutativity of $F$ and $G$, we write

$$
\begin{aligned}
& \max \left\{d\left(G\left(F\left(x_{2 n}, y_{2 n}\right)\right)\right), F\left(\alpha, \alpha^{\prime}\right)\right), \\
& \left.d\left(G\left(F\left(y_{2 n}, x_{2 n}\right)\right), F\left(\alpha^{\prime}, \alpha\right)\right)\right\} \\
& \leq \max \left\{d\left(G\left(G x_{2 n}\right), S(\alpha)\right), d\left(G\left(G y_{2 n}\right), S\left(\alpha^{\prime}\right)\right)\right\} .
\end{aligned}
$$

Letting $n \rightarrow+\infty$, using the continuity of $G$, we obtain

$$
\begin{aligned}
& \max \left\{d\left(G(\alpha), F\left(\alpha, \alpha^{\prime}\right)\right), d\left(G\left(\alpha^{\prime}\right), F\left(\alpha^{\prime}, \alpha\right)\right)\right\} \leq \\
& \max \left\{d(G(\alpha), S(\alpha)), d\left(G\left(\alpha^{\prime}\right), S\left(\alpha^{\prime}\right)\right)\right\} .
\end{aligned}
$$

Looking at (20), we deduce that

$$
\max \left\{d\left(G(\alpha), F\left(\alpha, \alpha^{\prime}\right)\right), d\left(G\left(\alpha^{\prime}\right), F\left(\alpha^{\prime}, \alpha\right)\right)\right\}=0 .
$$

Therefore,

$$
d\left(G(\alpha), F\left(\alpha, \alpha^{\prime}\right)\right)=0 \quad \text { and } \quad d\left(G\left(\alpha^{\prime}\right), F\left(\alpha^{\prime}, \alpha\right)\right)=0 .
$$

Consequently

$$
G(\alpha)=F\left(\alpha, \alpha^{\prime}\right) \quad \text { and } \quad G\left(\alpha^{\prime}\right)=F\left(\alpha^{\prime}, \alpha\right) .
$$

By the same way, we get

$$
S(\alpha)=F\left(\alpha, \alpha^{\prime}\right) \text { and } S\left(\alpha^{\prime}\right)=F\left(\alpha^{\prime}, \alpha\right) .
$$

Finally, combining (20), (23) and (24), we deduce that $\left(\alpha, \alpha^{\prime}\right)$ is a coupled coincidence point of $F, G$ and $S$.

\section{Remark 2}

Taking $G=S=I_{X}$ (the identity mapping of $X$ ) in Theorem 21, we obtain [7, Theorem 2].

Taking $G=S=I_{X}$ in Theorem 22, we obtain [7, Theorem 3].

Taking $S=G$, we get the following:

Corollary 21 Let $(X, \preceq)$ be a partially ordered set and suppose that there exists a metric $d$ on $X$ such that $(X, d)$ is a complete metric space. Let $G: X \rightarrow X$ be a continuous mapping and $F: X \times X \rightarrow X$ be a mapping having the mixed G-monotone property on $X$. Suppose that

$$
\begin{aligned}
& \varphi(d(F(x, y), F(u, v))) \leq \varphi(\max \{d(G x, G u), d(G y, G v)\}) \\
& -\phi(\max \{d(G x, G u), d(G y, G v)\}),
\end{aligned}
$$

for all $x, y, u, v \in X$ with $G(x) \preceq G(u)$ or $G(x) \succeq G(u)$ and $G(y) \succeq G(v)$ or $G(y) \preceq G(v)$, where $\varphi, \phi \in \mathscr{F}$. Assume that $\overline{F(} X \times X) \subseteq G(X) \cap G(X)$ and assume that

(I)F is continuous or assumptions (i) - (ii) of

Theorem 22 hold with $G$ non-decreasing.

(II)F commutes with $G$.

If there exist $x_{0}, y_{0}$ such that

$$
\left\{\begin{array}{l}
G\left(x_{0}\right) \preceq F\left(x_{0}, y_{0}\right) ; \\
G\left(y_{0}\right) \succeq F\left(y_{0}, x_{0}\right),
\end{array}\right.
$$

then there exist $x, y \in X$ such that

$$
G(x)=F(x, y) \quad \text { and } \quad G(y)=F(y, x),
$$

\section{Applications to nonlinear integral equations}

Let $X=C([0, T], \mathbb{R})$ be the set of all continuous functions $u:[0, T] \rightarrow \mathbb{R}, T>0$, and $G: X \rightarrow X$ is a given mapping. We endow $X$ with the metric $d(u, v)=\max _{t \in[0, T]}|u(t)-v(t)|$ for $u, v \in X$.

This space can be equipped with a partial order given by

$$
x, y \in X, \quad x \preceq y \Leftrightarrow x(t) \leq y(t), \quad \text { for any } t \in[0, T] .
$$

In $X \times X$ we define the following partial order

$(x, y),(u, v) \in X \times X, \quad(x, y) \preceq(u, v) \Leftrightarrow x \preceq u$ and $y \succeq v$.

In [10] it is proved that $(X, \preceq)$ satisfies assumptions $(i)$ and (ii) of Theorem 22.

Consider the system of integral equations:

$\left\{\begin{array}{l}G u(t)=\int_{0}^{T} k(t, s) f(s, u(s), v(s)) d s \\ G v(t)=\int_{0}^{T} k(t, s) f(s, v(s), u(s)) d s\end{array}\right.$ 
where the functions $k:[0, T] \times[0, T] \rightarrow[0,+\infty[$ and $f:[0, T] \times \mathbb{R} \times \mathbb{R} \rightarrow[0,+\infty[$ are two continuous functions satisfying the following conditions:

$(H 1)$

$$
\sup _{t \in[0, T]} \int_{0}^{T} k(t, s) d s \leq 1 .
$$

(H2) For all $s, b \in[0, T], u, v \in X$

$G u \preceq G v, \Rightarrow f(s, u(s), b) \leq f(s, v(s), b)$

$G u \preceq G v, \Rightarrow f(s, b, u(s)) \geq f(s, b, v(s))$.

(H3) For all $x, y, u, v \in X$ such that $G x \preceq G u$ and $G y \succeq G v$ we have

$$
\begin{aligned}
& |f(s, x(s), y(s))-f(s, u(s), v(s))| \leq \\
& \ln \left[1+(\max \{|G x(s)-G u(s)|,|G y(s)-G v(s)|\})^{2}\right] .
\end{aligned}
$$

(H4) There exist $\alpha, \beta \in X$ such that for all $t \in[0, T]$ we have

$$
\left\{\begin{array}{l}
G \alpha(t) \leq \int_{0}^{T} k(t, s) f(s, \alpha(s), \beta(s)) d s \\
G \beta(t) \leq \int_{0}^{T} k(t, s) f(s, \beta(s), \alpha(s)) d s .
\end{array}\right.
$$

Now, we shall prove the following result.

Theorem 31Suppose that $G: X \rightarrow X$ is a non-decreasing continuous mapping. Suppose also that (H1)-(H4) hold. Then (26) has a solution.

Proof. We introduce the operator $F: X \times X \rightarrow X$ defined by

$F(u, v)(t)=\int_{0}^{T} k(t, s)[f(s, u(s), v(s)) d s$

for all $u, v \in X$ and $t \in[0, T]$.

From (H2) it follows directly that $F$ has the mixed $G$ monotone property.

Let $u, v \in X$ such that $G(x) \preceq G(u)$ and $G(y) \succeq G(v)$. We have

$$
\begin{aligned}
& d(F(x, y), F(u, v))=\max _{t \in[0, T]}|F(x, y)(t)-F(u, v)(t)| \\
& \leq \max _{t \in[0, T]} \int_{0}^{T} k(t, s) \mid f(s, x(s), y(s))-f(s, u(s), v(s) \mid d s .
\end{aligned}
$$

Using (H3) we get

$$
\begin{gathered}
d(F(x, y), F(u, v)) \leq \max _{t \in[0, T]} \int_{0}^{T} k(t, s) \\
\ln \left[1+(\max \{|G x(s)-G u(s)|,|G y(s)-G v(s)|\})^{2}\right] d s \leq \\
\max _{t \in[0, T]} \int_{0}^{T} k(t, s) \ln \left[1+(\max \{d(G x, G u), d(G y, G v)\})^{2}\right] d s \\
\leq \ln \left[1+(\max \{d(G x, G u), d(G y, G v)\})^{2}\right] \times \\
\max _{t \in[0, T]} \int_{0}^{T} k(t, s) d s .
\end{gathered}
$$

From (H1), we obtain

$$
d(F(x, y), F(u, v)) \leq
$$

$\ln \left[(\max \{d(G x, G u), d(G y, G v)\})^{2}+1\right]$

which implies that

$$
\begin{aligned}
& (d(F(x, y), F(u, v)))^{2} \leq \\
& \left(\ln \left[(\max \{d(G x, G u), d(G y, G v)\})^{2}+1\right]\right)^{2} .
\end{aligned}
$$

Then,

$$
\begin{aligned}
& (d(F(x, y), F(u, v)))^{2} \leq(\max \{d(G x, G u), d(G y, G v)\})^{2} \\
& -\left[(\max \{d(G x, G u), d(G y, G v)\})^{2}\right. \\
& \left.-\left(\ln \left[(\max \{d(G x, G u), d(G y, G v)\})^{2}+1\right]\right)^{2}\right] .
\end{aligned}
$$

Set $\varphi(t)=t^{2}$ and $\phi(t)=t^{2}-\ln \left(t^{2}+1\right)$. Clearly $\varphi$ and $\phi$ are altering distance functions and from the above inequality, we obtain

$$
\begin{gathered}
\varphi(d(F(x, y), F(u, v))) \leq \varphi(\max \{d(G x, G u), d(G y, G v)\}) \\
\quad-\phi((\max \{d(G x, G u), d(G y, G v)\}))
\end{gathered}
$$

for all $x, y, u, v \in X$ such that $G(x) \preceq G(u)$ and $G(y) \succeq G(v)$. Now, let $\alpha, \beta \in X$ be the functions given by (H4), then we have

$$
G(\alpha) \preceq F(\alpha, \beta) \quad \text { and } \quad F(\beta, \alpha) \preceq G(\beta) .
$$

Thus, we proved that all the required hypotheses of Corollary 21 are satisfied. Hence, $G$ and $F$ have a coupled coincidence point $(u, v) \in X \times X$, that is, $(u, v)$ is a solution of (26).

\section{References}

[1] R. P. Agarwal, M. A. El-Gebeily, D. O'Regan, Generalized contractions in partially ordered metric spaces, Appl. Anal., 87, 109-116 (2008).

[2] I. Altun, H. Simsek, Some fixed point theorems on ordered metric spaces and application, Fixed Point Theory Appl., 2010, Article ID 621492, 17 pages (2010).

[3] T. G. Bhaskar, V. Lakshmikantham, Fixed point theorems in partially ordered metric spaces and applications, Nonlinear Anal., 65, 1379-1393 (2006).

[4] B. S. Choudhury, A. Kundu, A coupled coincidence point result in partially ordered metric spaces for compatible mappings, Nonlinear Anal., 73, 2524-2531 (2010).

[5] J. Harjani, K. Sadarangani, Fixed point theorems for weakly contractive mappings in partially ordered sets, Nonlinear Anal., 71, 3403-3410 (2008).

[6] J. Harjani, K. Sadarangani, Generalized contractions in partially ordered metric spaces and applications to ordinary differential equations, Nonlinear Anal., 72, 1188-1197 (2010). 
[7] J. Harjani, B. López, K. Sadarangani, Fixed point theorems for mixed monotone operators and applications to integral equations, Nonlinear Anal., 74, 1749-1760 (2011).

[8] V. Lakshmikantham, Lj. Ćirić, Coupled fixed point theorems for nonlinear contractions in partially ordered metric spaces, Nonlinear Anal., 70, 4341-4349 (2009).

[9] N. V. Luong, N. X. Thuan, Coupled fixed points in partially ordered metric spaces and application, Nonlinear Anal., 74, 983-992 (2011).

[10] J. J. Nieto, R. Rodŕiguez-López, Contractive mapping theorems in partially ordered sets and applications to ordinary differential equations, Order., 22, 223-239 (2005).

[11] J. J. Nieto, R. Rodŕiguez-López, Existence and uniqueness of fixed point in partially ordered sets and applications to ordinary differential equations, Acta Math. Sin., 23, 22052212 (2007)

[12] J. J. Nieto, R. L. Pouso, R. Rodŕiguez-López, Fixed point theorems in partially ordered sets, Proc. Amer. Soc., 132, 2505-2517 (2007).

[13] H. K. Nashine, B. Samet, Fixed point results for mappings satisfying $(\psi, \phi)$-weakly contractive condition in partially ordered metric spaces, Nonlinear Anal., 74, 2201-2209 (2011).

[14] A. C. M. Ran, M. C. B. Reurings, A fixed point theorem in partially ordered sets and some applications to metrix equations, Proc. Amer. Math. Soc., 132, 1435-1443 (2004).

[15] M. D. Rus, Fixed point theorems for generalized contractions in partially ordered metric spaces with semimonotone metric, Nonlinear Anal., 74, 1804-1813 (2011).

[16] B. Samet, Coupled fixed point theorems for a generalized Meir-Keeler contraction in partially ordered metric spaces, Nonlinear Anal., 72, 4508-4517 (2010).

[17] W. Shatanawi, Partially ordered cone metric spaces and coupled fixed point results, Comput. Math. Appl., 60, 25082515 (2010).

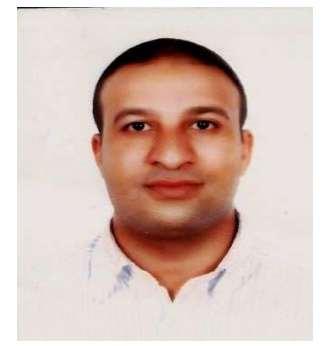

$\begin{array}{clc}\text { Habib } & \text { Yazidi is } \\ \text { an assistant } & \text { professor } & \text { at }\end{array}$ Department of Mathematics, Tunis College of Sciences and Techniques, University of Tunis. He received the $\mathrm{PhD}$ degree in Mathematics at Paris Est University, France. His research interests are in the areas of non-linear Analysis, PDE and fixed points theory. 